\title{
Impacto de los costos de calidad en la ejecución de los proyectos de construcción en Colombia ${ }^{1}$
}

\author{
César Augusto Silva Giraldo² \\ Corporación Universitaria Minuto de Dios - Uniminuto \\ csilvagiral@uniminuto.edu.co \\ Juan Sebastián Dugarte Mendoza ${ }^{3}$ \\ Corporación Universitaria Minuto de Dios - Uniminuto \\ mdugarte@uniminuto.edu.co \\ Aizar Mejía Jálabe ${ }^{4}$ \\ Corporación Universitaria Minuto de Dios - Uniminuto \\ amejiajalab@uniminuto.edu.co
}

Fecha de recepción: 22 de junio de 2018

Fecha de aprobación: 03 de septiembre de 2018

DOI: https://doi.org/10.21158/01208160.n0.2018.2017

Cómo citar este artículo / To reference this article / Comment citer cet article / Para citar este artigo:

Silva Giraldo, C. A.; Dugarte Mendoza, J. S. y Mejía Jálabe, A. (2018). Impacto de los costos de calidad en la ejecución de los proyectos de construcción en Colombia. Revista EAN, Edición especial, pp. 33-54. DOI: https://doi.org/10.21158/01208160. n0.2018.2017

\section{Resumen}

El impacto que genera el plan de gestión de calidad de un proyecto se ve reflejado en indicadores de medición elementales para la toma de decisiones. Es tal su importancia dentro del progreso de cualquier proyecto, que si se ejecuta de forma adecuada puede evitar la inclusión de valores adicionales y posibles reprocesos. Por ello, el presente artículo tiene por objetivo analizar el impacto de los costos de calidad en la ejecución de proyectos de construcción en Colombia. A través de una investigación de tipo de correlacional con enfoque cuantitativo, se desarrolló un estudio de caso a partir de ocho proyectos de construcción de edificaciones, que llevó a concluir que no es evidente la recuperación de los costos de calidad, en ninguna de las opciones analizadas, bajo los métodos de simulación de Monte Carlo y el análisis del valor ganado, por lo cual los costos deben ser presupuestados y tenidos en cuenta en las diversas etapas de ejecución de los proyectos.

\section{Palabras Clave}

Ejecución de proyectos, industria de la construcción - Colombia, industria de la construcción -aspectos económicos, control de calidad, toma de decisiones.

${ }^{1}$ Artículo derivado del proyecto de investigación Estudio del impacto de los costos de calidad en la ejecución de los proyectos, de la Corporación Universitaria Minuto de Dios (Uniminuto) Centro Regional Bucaramanga, Especialización en Gerencia de Proyecto.

2 Administrador de Empresas, Universidad Nacional Abierta y a Distancia UNAD. Especialista en Gestión de Proyectos, Universidad Nacional Abierta y a Distancia UNAD. MBA- Master en Dirección y Administración de Empresas, Universidad Rey Juan Carlos. MBA - Master especializado en Comercio Internacional, Cerem Business School. Maestrando en Paz, Desarrollo y Ciudadanía, Uniminuto. Doctorando en Ciencias Económicas y Administrativas, UCIMEXICO México. ORCID: https://orcid.org/0000-0002-8023-8531

${ }_{3}^{3}$ Ingeniero Mecánico, Universidad Pontificia Bolivariana. MBA - Magister en Administración de Empresas con Especialidad en Dirección de Proyectos, Universidad de Viña del Mar - Chile. Doctorando Dirección de Proyectos, Universidad Benito Juárez, México. ORCID: https://orcid.org/0000-0003-1149-0503

4 Ingeniero Industrial, Universidad Pontificia Bolivariana. Especialista en Gerencia de Proyectos, Universidad del Tolima. Magister en Administración, Universidad Santo Tomás de Aquino. USTA. Doctorando en Ciencias Económicas y Administrativas, UCIMEXICO, México. ORCID: https://orcid.org/0000-0002-9454-4513 


\title{
Impact of quality cost execution of construction projects in Colombia
}

\begin{abstract}
The impact which generates the planning of quality management projects is estimated through basic measurement indicators to take decisions in organizations. It is so relevant to know if during the execution of any project decisions have been appropriately taken to avoid the inclusion of added values and possible undesirable results. For this reason, this research paper is intended to analyze the impact of quality costs in the execution of construction projects in Colombia. This correlational investigation with a quantitative focus studied an actual case involving 8 other construction projects which concluded that there was no evidence that quality costs could be recovered in any case, using the Monte Carlo simulation methods and the analysis of added value, which clearly showed that costs had to be budgeted and included during different execution stages of projects.
\end{abstract}

key words: Project execution, construction economic sector-Colombia, construction industry-economic aspects, quality control, decision taking.

\section{Impact des coûts de qualité lors de l'exécution des projets de construction en Colombie}

Résumé. L'impact créé par le plan de gestion de la qualité d'un projet se reflète dans les indicateurs de mesure élémentaires pour la prise de décision. La gestion de la qualitè est fondamentale pour l'état d'avancement de tout projet et exécutée correctement elle permet d'éviter des frais supplémentaires et possible retards. Cet article tente d'analyser l'impact des coûts liés à la qualité de l'exécution des projets de construction en Colombie. Á partir d'une enquête quantitative corrélationnelle, une étude de cas a été réalisée portant sur huit projets de construction de bâtiments et conduisant à la conclusion que le recouvrement des coûts liés à la qualité n'est pas toujours assuré à la lumiére des alternatives prises en compte par les méthodes de simulation de Monte Carlo et d'analyse de la valeur acquise. Les coûts doivent impérativement être inscrits au Budget initial et pris en compte à différents stades de la mise en ceuvre du projet.

Mots clefs: Exécution de projets, industrie de la construction - Colombie, industrie de la construction - aspects économiques, contrôle de la qualité, prise de décision.

\section{Impacto dos custos de qualidade na execução dos projetos de construção na Colômbia}

Resumo. El impacto que gera o plano de gerenciamento de qualidade de um projeto se vê refletido em indicadores de medida elementares para a toma de decisões. É tal sua importância dentro do progresso de qualquer projeto, que se se executa de forma adequada pode evitar a inclusão de valores adicionais e possíveis reprocessos. Por isso, o presente artigo tem por objetivo analisar o impacto dos custos de qualidade na execução de projetos de construção na Colômbia. Através de uma pesquisa de tipo correlacional com enfoque quantitativo, desenvolveu-se um estudo de caso a partir de oito projetos de construção de edificações, que levou a concluir que não é evidente a recuperação dos custos de qualidade em nenhuma das opções analisadas, sob os métodos de simulação de Monte Carlo e a análise do valor ganho, pelo que os custos devem ser orçados e tidos em conta nas diversas etapas de execução dos projetos.

Palavras-chave: Execução de projetos, indústria da construção - Colômbia, indústria da construção - aspectos econômicos, controle de qualidade, toma de decisões. 


\section{Introducción}

os proyectos son formulados con el
propósito de satisfacer necesidades, solucionar problemas, generar oportunidades de inversión y crear productos o servicios, a partir de requisitos técnicos que posibilitan el desarrollo de conocimientos, habilidades y destrezas. Estas exigencias buscan que la formulación y la ejecución planteada en la metodología se articulen con los objetivos y resultados esperados (Project Management Institute [PMI], 2017).

Para formular un proyecto, es necesario realizar un análisis de los interesados e involucrados, mediante un estado del arte que permita identificar las lecciones aprendidas, el alcance, el costo y el tiempo, lo cual marca las restricciones de los proyectos para su ejecución. Estas usualmente son establecidas por las organizaciones a través de la estructura y el marco de gobernanza, con el objetivo de determinar los posibles riesgos y la calidad de los procesos (PMI, 2017).

Por lo anterior, para conseguir la operatividad de manera eficiente y eficaz del proyecto, se requiere que el director promueva la creación de un lazo de responsabilidad que facilite la rendición de cuentas, la autoridad en la organización, el poder de influencia, las competencias y el liderazgo en la toma de decisión, ya que esta adquiere relevancia en la ejecución de los proyectos debido a que permite definir los procesos, las actividades y los recursos que se articulan con los objetivos mediante la producción de entregables definidos como productos tangibles o intangibles (PMI, 2017).

La toma de decisiones se orienta alrededor de los procesos de calidad, que son establecidos en el plan de gestión, donde se acuerdan los requerimientos mínimos que incluyen técnicas y herramientas para la inspección, el control y la vigilancia a partir de las auditorías e inspecciones. Por ello, el plan de gestión de calidad, además de incluir el desarrollo y cumplimiento de normas y especificaciones, también requiere una articulación de la política de calidad de la organización, con el fin de generar una integralidad con los lineamientos y las estrategias propuestos desde la alta dirección (Lledó y Rivola, 2007).

Teniendo en cuenta lo mencionado, cabe resaltar que el impacto que genera el plan de gestión de calidad se ve reflejado en indicadores de medición elementales para la toma de decisiones, que son la rentabilidad, la articulación de los procesos o el análisis y el control total de los costos asociados. Es tal su importancia dentro del progreso de cualquier proyecto que, si se ejecutan de forma adecuada, puede evitar la inclusión de valores adicionales y posibles reprocesos. A partir, entonces, de la premisa del plan de gestión de calidad, se considera necesario analizar y desarrollar técnicas o estrategias alineadas con las exigencias de las organizaciones que aporten al desarrollo integral de cada proyecto (Gido y Clements, 2012).

Cabe resaltar que una de las principales exigencias para el desarrollo integral de los proyectos se encuentra relacionada con el presupuesto que establece el sponsor para cada propuesta, ya que en la mayoría de casos suele ser limitado o restringido. Por tanto, se contempla el factor diferenciador entre la manera costosa y la forma provechosa de alcanzar la calidad. En este sentido, los costos en este aspecto permiten a las organizaciones precisar las fuerzas y las debilidades de un sistema de gestión de calidad (Ahmed, Aoieong, Tang y Zheng, 2005).

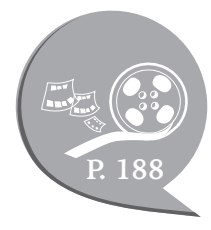


Los costes de calidad son definidos como los gastos que se presentan por la corrección de los fallos dados en la verificación de procesos o actividades. Por tanto, en los proyectos se debe desarrollar un alcance adecuado, basado en los valores y los tiempos reales que se establecen a partir de una línea base y las diferentes variables que se pueden presentar en la realización del plan (Gracia y Dzul, 2007). El análisis de costos parte del presupuesto inicial y la línea del tiempo que muestra el panorama de la ejecución, de este modo se consigue comparar los resultados con supuestos, incluso los costos de calidad que afectan el valor final (PMI, 2017).

La gestión de costos en los proyectos en general se ocupa del costo de los recursos necesarios para desarrollar actividades, sin tener el cálculo de los valores repetidos posteriores, generados por el producto, servicio o resultados específicos. Por lo anterior, es necesario establecer la medición y evaluación de los costos de calidad, que van a estar inmersos en diversos procesos y acciones involucrados directamente en el sistema de calidad, llegando a convertirse en costos recurrentes sobre los que se debe desplegar un seguimiento y control. Hace algunos años estos factores relacionados con la gestión de calidad no se tenían en cuenta, pero, en la actualidad, las organizaciones han venido acogiendo técnicas de certificación en diversas normas que impactan claramente los costos de ejecución de los proyectos (Campanella, 1997).

En síntesis, la implementación de las políticas de gestión de calidad requieren un control adecuado de la información para favorecer la toma de decisiones frente a las medidas necesarias que contrarresta las anomalías, las desviaciones o los defectos que se van a reflejar en el incremento de los valores, de esta manera se puede llegar a la prevención de los errores y la eliminación temprana de las fallas en actividades básicas incluidas en la gestión de la calidad donde se logra presentar un aumento en los costos (Climent, 2004).

La gestión que se genera sobre los costos de calidad va a permitir a la dirección determinar los productos defectuosos, los problemas por falta de condiciones y los potenciales ahorros que se puedan presentar por la calidad en los procesos. De igual manera, los directores pueden realizar una valoración de la rentabilidad de la inversión, con lo cual se convierten en un elemento fundamental en la planificación estratégica de la empresa en relación con el proyecto en ejecución. 


\section{Metodología}

T a investigación se desarrolló por medio Lde un tipo correlacional con un enfoque cuantitativo. Según Salkind (citado por Bernal, 2016), el propósito de estos estudios es mostrar o examinar la relación existente entre diversas variables. Para ello, se recurre al estudio de caso como método de investigación, que permite estudiar a profundidad una unidad de análisis dentro de un universo poblacional a partir de un tema específico. Por tanto, para el caso de estudio, se tomaron los costos de ocho obras de construcción de edificaciones ejecutadas con características similares. Posteriormente, se establecieron las actividades que hacían referencia a acabados y así inferir objetivamente si los valores de calidad afectan en menor o mayor proporción los costos totales de ejecución de los proyectos (Bernal, 2016).

El estudio realizado contó con el apoyo de la Corporación Universitaria Minuto de Dios (Uniminuto) Centro Regional Bucaramanga y la Especialización en Gerencia de Proyectos. Se inició a partir de un análisis de los procesos estocásticos, haciendo uso del método de simulación de Monte Carlo por medio del software Risk Simulator, con el propósito de establecer los riesgos de la obra frente al presupuesto, al no tener presente los costos de calidad.

Los procesos estocásticos se definen como una colección de variables aleatorias parametrizadas, donde las variables llegan a tomar valores en conjunto de acuerdo con el espacio de los estados de las variables (Rincón, 2012). Lo descrito conlleva la utilización del método de Monte Carlo. Este procedimiento permite la simulación estadística y el valor final de una serie de sucesos sujetos a variabilidad (Zapata, 2011).
Por tanto, el diseño del estudio está dado por los valores de los proyectos teniendo presente la variable de tiempo, es decir, se estableció una serie de planes desarrollados en el sector de la construcción según la etapa que corresponde a acabados que es donde normalmente se presenta una variabilidad en los costos.

A los procesos y las actividades que hacen parte de cada una de las etapas se les ejecuta un ajuste de distribución de las variables de tiempo y costo. Posteriormente, se realiza la simulación a través del software Risk Simulator teniendo en cuenta aquellas acciones que pueden verse afectadas por costos de calidad que permitirá el cálculo del valor medio y la variabilidad para el conjunto entre todos los proyectos desarrollados en un periodo determinado.

Al finalizar el proceso, se aplicó el método del gestión del valor ganado (EVM 'earned value management'), que es el costo presupuestado de lo realizado en comparación con la variable de tiempo; es decir, si el desarrollo de lo ejecutado de la actividad coincide con lo que se predijo inicialmente, el valor ganado debe coincidir con el costo planificado. Al incluir la variable del costo de calidad en la ejecución de las actividades inmersas de acuerdo con el plan de gestión de calidad de la organización y que afecta la obra, este podría llegar a indicar una variabilidad en el presupuesto planeado, determinando de igual manera que el proyecto se ejecuta según los tiempos previstos. En caso contrario, se mostraría adelanto o atraso y la influencia de los costos de calidad con su respectivo impacto desde un punto de vista cuantitativo, el mismo que se demuestra en los resultados y conclusiones de la investigación (Navarro, 2006). 


\section{Resultados}

Dara la ejecución de la investigación, Pe estableció un conjunto de etapas ajustadas a las metodologías de análisis de incertidumbre de proyectos, tomando como caso de estudio ocho proyectos de construcción de edificaciones, en busca de determinar en ellos la varianza natural del proyecto a partir de la sumatoria de las varianzas de las actividades incluidas en la ruta crítica, específicamente desde el punto de vista del tiempo y costo. Además, estos valores de varianza son comprensibles para el ejecutor de la obra, desde lo numérico y gráfico a través de la EVM y el desarrollo de las curvas S estocásticas, respectivamente (Barraza, Back y Mata, 2004; Hazır, 2015).
En concordancia con lo anterior, se desarrolla el siguiente procedimiento para el análisis de los costos de calidad. En primer lugar, se realiza un estudio de la base de datos generada por Batselier y Vanhoucke (2015) y Vanhoucke, Coelho y Batselier (2016), con el objetivo de determinar un conjunto de obras con acciones iguales y ejecutadas bajo contextos similares. Además, se busca que presenten sus respectivos valores de costos y tiempos causados, así como la ruta crítica definida a partir de las dependencias existentes entre actividades, tras lo cual se obtiene un total de ocho proyectos desarrollados en el sector de la construcción, específicamente en la etapa de acabados, como se observa en la tabla 1. Este paso se realizó con apoyo de la herramienta Microsoft Project 2016 y Microsoft Excel.

Tabla 1. Listado de actividades, clasificación y organización

\begin{tabular}{|c|c|c|c|c|c|}
\hline \multicolumn{3}{|c|}{ Código de proyecto } & \multirow{2}{*}{ Clasificación } & \multirow{2}{*}{ Precedencias } & \multirow{2}{*}{$\begin{array}{l}\text { Actividad en } \\
\text { ruta crítica }\end{array}$} \\
\hline Actividad & ID & EDT & & & \\
\hline Trabajos de acabados en casas residenciales & & 1 & & & \\
\hline Inicio de etapa de acabados & & 1.1 & & & \\
\hline Sistema de ventilación & 1 & 1.1 .1 & determinística & & SI \\
\hline san / cv, $1 .^{\mathrm{a}}$ etapa & 2 & 1.1 .2 & determinística & $1 \mathrm{FS}$ & SI \\
\hline Red eléctrica, $1 .^{a}$ etapa & 3 & 1.1 .3 & determinística & $2 \mathrm{FS}$ & SI \\
\hline Sistema de hidráulico & 4 & 1.1 .4 & determinística & $3 \mathrm{FS}$ & NO \\
\hline Aislamiento de techo & 5 & 1.1 .5 & determinística & $3 \mathrm{FS}$ & SI \\
\hline Aplicaciones de yeso & 6 & 1.1 .6 & estocástica & $5 \mathrm{FS}$ & SI \\
\hline Fase 2 & & 1.2 & & & \\
\hline Instalación de red de gas & 7 & 1.2 .1 & determinística & $6 \mathrm{FS}$ & SI \\
\hline $\operatorname{san} / \mathrm{cv}, 2 .^{\mathrm{a}}$ etapa & 25 & 1.2 .2 & determinística & $6 \mathrm{FS}$ & SI \\
\hline Sistema de ventilación & 8 & 1.2 .3 & determinística & 7FS;25FS & $\mathrm{NO}$ \\
\hline Aislamiento de piso & 9 & 1.2 .4 & determinística & 7FS;25FS & SI \\
\hline Enchape metálico & 10 & 1.2 .5 & determinística & 9FS & SI \\
\hline
\end{tabular}


Tabla 1. Listado de actividades, clasificación y organización (Continuación)

\begin{tabular}{|c|c|c|c|c|c|}
\hline \multicolumn{3}{|c|}{ Código de proyecto } & \multirow{2}{*}{ Clasificación } & \multirow{2}{*}{ Precedencias } & \multirow{2}{*}{$\begin{array}{l}\text { Actividad en } \\
\text { ruta crítica }\end{array}$} \\
\hline Actividad & ID & EDT & & & \\
\hline Pintura de interior & 11 & 1.2 .6 & determinística & 10FS & NO \\
\hline Red eléctrica, 2. ${ }^{a}$ etapa & 12 & 1.2.7 & determinística & 10FS & SI \\
\hline Instalación de servicios (energía, agua, gas) & 13 & 1.2 .8 & estocástica & $12 \mathrm{FS}$ & SI \\
\hline Fase 3 & & 1.3 & & & \\
\hline $\mathrm{san} / \mathrm{cv}, 3 .^{\mathrm{a}}$ etapa & 14 & 1.3 .1 & determinística & $13 \mathrm{FS}$ & SI \\
\hline Piso & 15 & 1.3 .2 & determinística & $14 \mathrm{FS}$ & SI \\
\hline Escaleras & 16 & 1.3 .3 & determinística & $15 \mathrm{FS}$ & SI \\
\hline Carpintería interior & 17 & 1.3 .4 & determinística & $16 \mathrm{FS}$ & SI \\
\hline Acabados de cocina & 18 & 1.3 .5 & determinística & $17 \mathrm{FS}$ & SI \\
\hline Acabado final & & 1.4 & & & \\
\hline Finalización de sistema de ventilación & 19 & 1.4 .1 & estocástica & $18 \mathrm{FS}$ & SI \\
\hline Finalización de sistema eléctrico & 20 & 1.4 .2 & estocástica & $18 \mathrm{FS}$ & SI \\
\hline Finalización de san / cv & 21 & 1.4 .3 & estocástica & 19FS;20FS & SI \\
\hline Finalización de piso & 26 & 1.4 .4 & estocástica & $19 \mathrm{FS}$ & SI \\
\hline Finalización de acabados de yeso & 24 & 1.4 .5 & estocástica & 19FS;20FS & SI \\
\hline
\end{tabular}

Fuente. Elaboración propia.

Por otra parte, se establece la ruta crítica del proyecto, a partir de las precedencias entregadas por la base de datos mencionada. De esta forma, se comprende la relación entre actividades y por tanto se identifican aquellas generadoras de impacto desde el punto de vista del tiempo y costo, en caso de presentar un reproceso por concepto de fallas o falencias en aspectos relacionados con la calidad.

Una vez identificadas las actividades de la ruta crítica, se procede a realizar la estimación de la curva de probabilidad sobre aquellas definidas como "estocásticas", con ello se busca reconocer su comportamiento y así permitir la observación de su valor medio, varianza y desviación estándar real, que se observa en las figuras 1, 2 y 3 . El proceso de selección de la distribución se realizó de acuerdo con el valor del factor $P$, en el que un resultado cercano a 1 determina un valor de ajuste conveniente de la curva de probabilidad respecto de la variable analizada; este paso fue realizado con apoyo de la herramienta Risk Simulator.

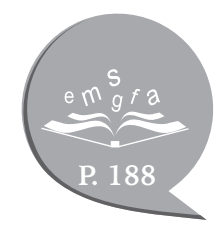


Figura 1. Resultado análisis de ajuste de distribución simple, variable «Pintura interior 1.2.6 costo»

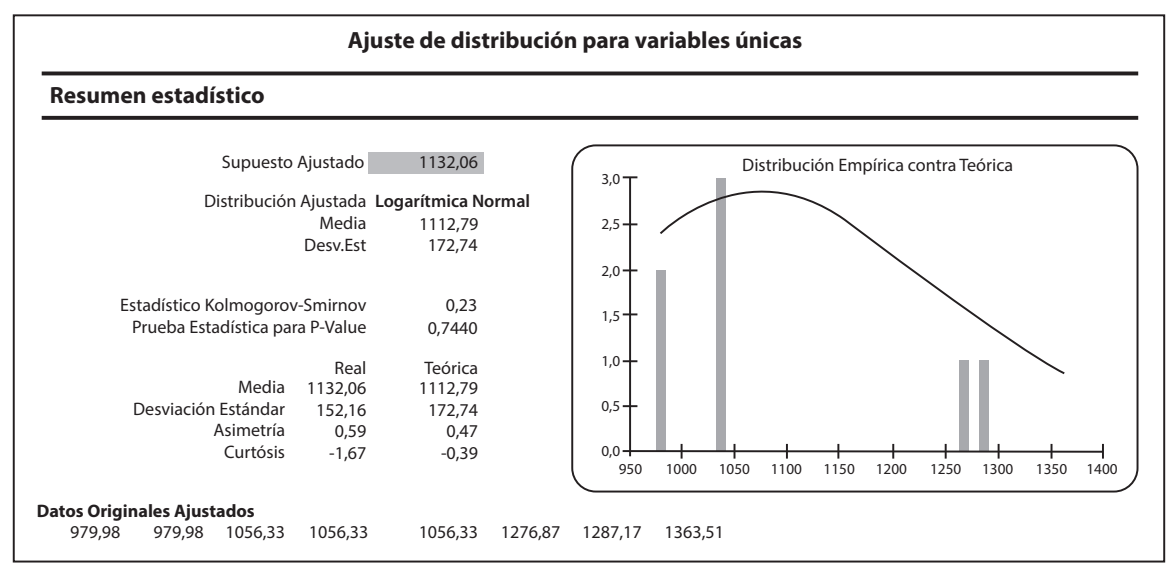

Fuente. Elaboración propia.

Figura. 2. Resultado análisis de ajuste de distribución simple, variable «Escaleras 1.3.3 costo»

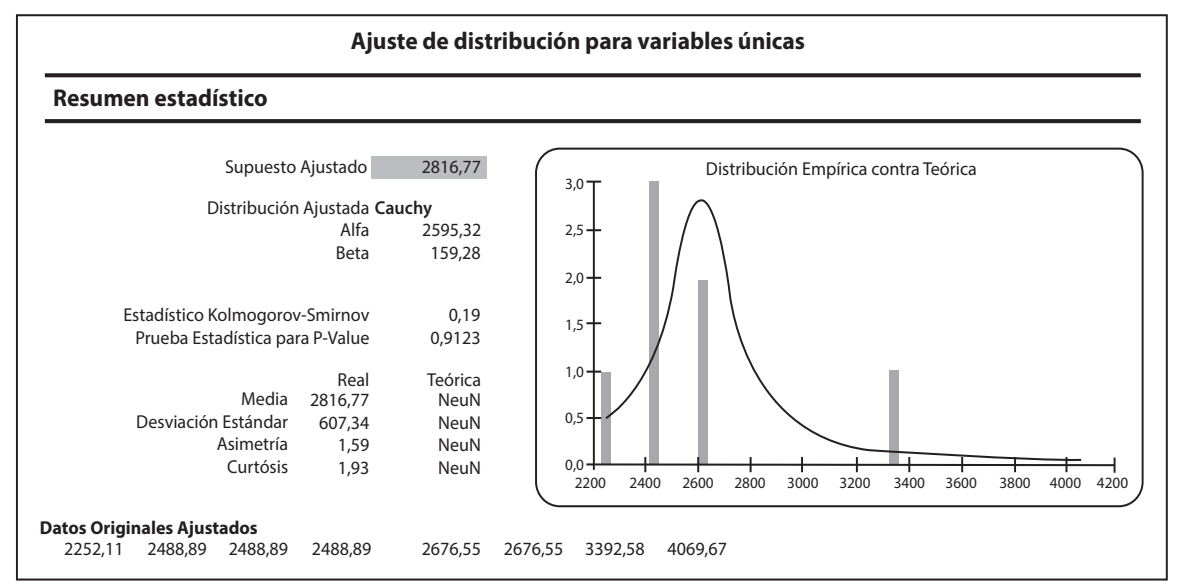

Fuente. Elaboración propia.

Figura 3. Resultado análisis de ajuste de distribución simple, variable «Finalización de acabados de yeso 1.4.5 costo»

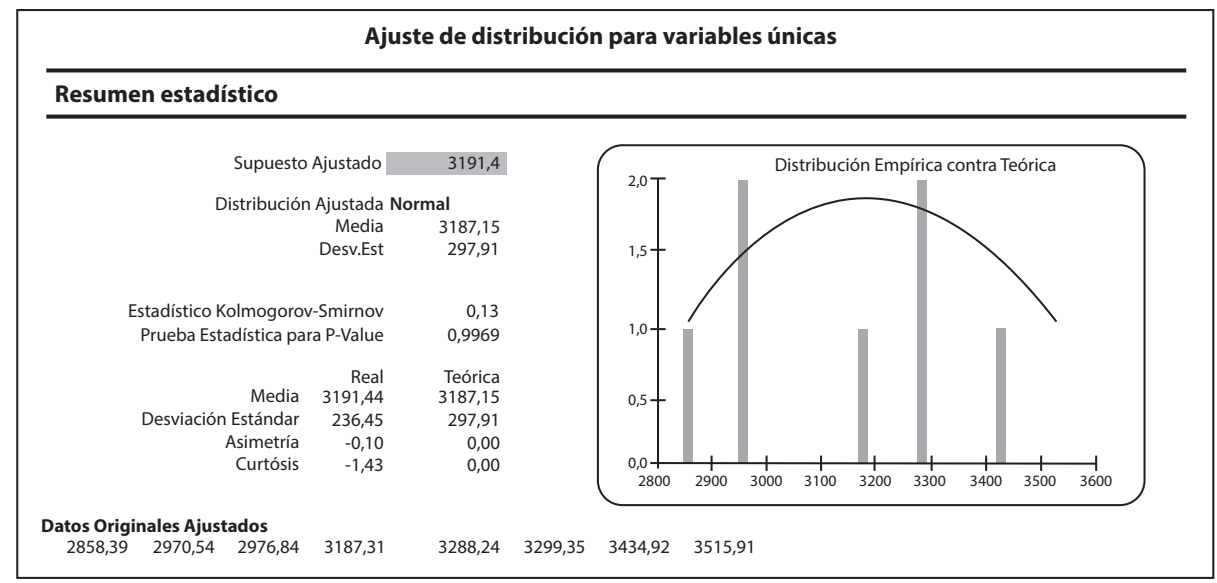

Fuente. Elaboración propia. 
En paralelo al paso anterior, se definen aquellas acciones generadoras de reprocesos por motivos de falencias o fallas desde el punto de vista de la calidad, considerando el reproceso como la necesidad de deshacer la tarea y volver a realizarse debido a imperfecciones que afecten la percepción de la calidad por parte del cliente final. Esta labor es realizada con apoyo de un comité de expertos, que define las actividades que se observan en la tabla 2.

Tabla 2. Listado de actividades clasificadas con los valores de media y desviación estándar calculados y con identificación de actividades susceptibles a reprocesos durante la ejecución

\begin{tabular}{|c|c|c|c|c|c|c|c|c|}
\hline \multicolumn{2}{|l|}{ Proyecto } & \multicolumn{3}{|c|}{ Tiempo (días) } & \multicolumn{3}{|c|}{ Costo (EUR) } & \multirow{2}{*}{$\begin{array}{l}\text { Actividades } \\
\text { con posibles } \\
\text { reprocesos }\end{array}$} \\
\hline Actividad & EDT & $\begin{array}{l}\text { Tipo de } \\
\text { actividad }\end{array}$ & Media & $s^{2}$ & $\begin{array}{l}\text { Tipo de } \\
\text { actividad }\end{array}$ & Media & $\sigma^{2}$ & \\
\hline $\begin{array}{l}\text { Trabajos de acabados } \\
\text { en casas residenciales }\end{array}$ & 1 & & 90,25 & 17,09 & & $\begin{array}{l}54360,83 \\
\text { EUR }\end{array}$ & $\begin{array}{l}2659,78 \\
\text { EUR }\end{array}$ & \\
\hline $\begin{array}{l}\text { Inicio de etapa de } \\
\text { acabados }\end{array}$ & 1.1 & & 28,75 & 2,31 & & $\begin{array}{l}8661,79 \\
\text { EUR }\end{array}$ & 380,9 EUR & \\
\hline Sistema de ventilación & 1.1 .1 & determinista & 5 & 0 & determinista & 772,50 EUR & 0 & \\
\hline san / cv, 1. ${ }^{\mathrm{a}}$ etapa & 1.1 .2 & determinista & 5 & 0 & determinista & $\begin{array}{l}1288,55 \\
\text { EUR }\end{array}$ & 0 & \\
\hline Red eléctrica, $1 .^{\text {a }}$ etapa & 1.1 .3 & determinista & 5 & 0 & estocástica & 853,57 EUR & 26,27 EUR & $\mathrm{X}$ \\
\hline Sistema de hidráulico & 1.1 .4 & determinista & 5 & 0 & determinista & 960,00 EUR & 0 & $\mathrm{X}$ \\
\hline Aislamiento de techo & 1.1 .5 & determinista & 5 & 0 & estocástica & $\begin{array}{l}2393,58 \\
\text { EUR }\end{array}$ & $\begin{array}{l}177,35 \\
\text { EUR }\end{array}$ & $\mathrm{X}$ \\
\hline Aplicaciones de yeso & 1.1 .6 & estocástica & 8,75 & 2,31 & estocástica & $\begin{array}{l}2393,58 \\
\text { EUR }\end{array}$ & $\begin{array}{l}177,35 \\
\text { EUR }\end{array}$ & \\
\hline Fase 2 & 1.2 & & 21,50 & 1,41 & & $\begin{array}{l}\text { 13.138,05 } \\
\text { EUR }\end{array}$ & $\begin{array}{c}603,16 \\
\text { EUR }\end{array}$ & \\
\hline $\begin{array}{l}\text { Instalación de red de } \\
\text { gas }\end{array}$ & 1.2 .1 & determinista & 5 & 0 & determinista & $\begin{array}{l}2231,25 \\
\text { EUR }\end{array}$ & 0 & \\
\hline san / cv, 2. ${ }^{\mathrm{a}}$ etapa & 1.2 .2 & determinista & 5 & 0 & determinista & $\begin{array}{l}1932,83 \\
\text { EUR }\end{array}$ & 0 & \\
\hline Sistema de ventilación & 1.2 .3 & determinista & 5 & 0 & determinista & $\begin{array}{l}1158,75 \\
\text { EUR }\end{array}$ & 0 & \\
\hline Aislamiento de piso & 1.2 .4 & determinista & 5 & 0 & estocástica & $\begin{array}{c}3128,73 \\
\text { EUR }\end{array}$ & $\begin{array}{c}230,55 \\
\text { EUR }\end{array}$ & $\mathrm{X}$ \\
\hline Enchape metálico & 1.2 .5 & determinista & 5 & 0 & estocástica & $\begin{array}{l}\text { 1955,31 } \\
\text { EUR }\end{array}$ & $\begin{array}{c}181,05 \\
\text { EUR }\end{array}$ & \\
\hline Pintura de interior & 1.2 .6 & determinista & 5 & 0 & estocástica & $\begin{array}{l}1132,06 \\
\text { EUR }\end{array}$ & $\begin{array}{l}152,16 \\
\text { EUR }\end{array}$ & \\
\hline Red eléctrica, 2. ${ }^{\text {a }}$ etapa & 1.2 .7 & determinista & 5 & 0 & estocástica & $\begin{array}{c}1280,36 \\
\text { EUR }\end{array}$ & 39,41 EUR & $X$ \\
\hline $\begin{array}{l}\text { Instalación de } \\
\text { servicios (energía, } \\
\text { agua, gas) }\end{array}$ & 1.2 .8 & estocástica & 1,50 & 1,41 & determinista & 318,75 EUR & 0 & \\
\hline
\end{tabular}


Tabla 2. Listado de actividades clasificadas con los valores de media y desviación estándar calculados y con identificación de actividades susceptibles a reprocesos durante la ejecución (Continuación)

\begin{tabular}{|c|c|c|c|c|c|c|c|c|}
\hline \multicolumn{2}{|l|}{ Proyecto } & \multicolumn{3}{|c|}{ Tiempo (días) } & \multicolumn{3}{|c|}{ Costo (EUR) } & \multirow{2}{*}{$\begin{array}{l}\text { Actividades } \\
\text { con posibles } \\
\text { reprocesos }\end{array}$} \\
\hline Actividad & EDT & $\begin{array}{l}\text { Tipo de } \\
\text { actividad }\end{array}$ & Media & $s^{2}$ & $\begin{array}{l}\text { Tipo de } \\
\text { actividad }\end{array}$ & Media & $\sigma^{2}$ & \\
\hline Fase 3 & 1.3 & & 25 & 0 & & $\begin{array}{c}13647,06 \\
\text { EUR }\end{array}$ & $\begin{array}{l}989,27 \\
\text { EUR }\end{array}$ & \\
\hline san / cv, 3. ${ }^{a}$ etapa & 1.3 .1 & determinista & 5 & 0 & determinista & $\begin{array}{c}\text { 3221,38 } \\
\text { EUR }\end{array}$ & 0 & \\
\hline Piso & 1.3 .2 & determinista & 5 & 0 & estocástica & $\begin{array}{c}2085,82 \\
\text { EUR }\end{array}$ & $\begin{array}{l}153,70 \\
\text { EUR }\end{array}$ & \\
\hline Escaleras & 1.3 .3 & determinista & 5 & 0 & estocástica & $\begin{array}{c}2816,77 \\
\text { EUR }\end{array}$ & $\begin{array}{c}607,34 \\
\text { EUR }\end{array}$ & \\
\hline Carpintería interior & 1.3 .4 & determinista & 5 & 0 & estocástica & $\begin{array}{c}1698,09 \\
\text { EUR }\end{array}$ & $\begin{array}{c}228,24 \\
\text { EUR }\end{array}$ & \\
\hline Acabados de cocina & 1.3 .5 & determinista & 5 & 0 & determinista & $\begin{array}{l}3825,00 \\
\text { EUR }\end{array}$ & 0 & $\mathrm{X}$ \\
\hline Acabado final & 1.4 & & 15,00 & 13,36 & & $\begin{array}{c}18913,93 \\
\text { EUR }\end{array}$ & $\begin{array}{l}686,38 \\
\text { EUR }\end{array}$ & \\
\hline $\begin{array}{l}\text { Finalización de } \\
\text { sistema de ventilación }\end{array}$ & 1.4 .1 & estocástica & 7,50 & 2,67 & determinista & $\begin{array}{c}1931,25 \\
\text { EUR }\end{array}$ & 0 & \\
\hline $\begin{array}{l}\text { Finalización de } \\
\text { sistema eléctrico }\end{array}$ & 1.4 .2 & estocástica & 7,50 & 2,67 & estocástica & $\begin{array}{l}2133,94 \\
\text { EUR }\end{array}$ & 65,68 EUR & $\mathrm{X}$ \\
\hline $\begin{array}{l}\text { Finalización de san / } \\
\mathrm{cv}\end{array}$ & 1.4 .3 & estocástica & 7,50 & 2,67 & determinista & $\begin{array}{l}6442,75 \\
\text { EUR }\end{array}$ & 0 & \\
\hline Finalización de piso & 1.4 .4 & estocástica & 7,50 & 2,67 & estocástica & $\begin{array}{c}5214,55 \\
\text { EUR }\end{array}$ & $\begin{array}{l}384,25 \\
\text { EUR }\end{array}$ & $\mathrm{X}$ \\
\hline $\begin{array}{l}\text { Finalización de } \\
\text { acabados de yeso }\end{array}$ & 1.4 .5 & estocástica & 7,50 & 2,67 & estocástica & $\begin{array}{c}\text { 3191,44 } \\
\text { EUR }\end{array}$ & $\begin{array}{l}236,45 \\
\text { EUR }\end{array}$ & \\
\hline
\end{tabular}

\section{Fuente. Elaboración propia.}

Con los valores calculados en la tabla 2, se procedió a determinar las condiciones mínimas, medias y máximas de variación, aplicando como base teórica el concepto de la varianza natural de un proyecto, la cual indica que toda actividad susceptible a cambios de sus atributos cuantitativos por factores internos o externos a la obra mostrará un cambio explicable a través de las desviaciones estándar. Por ende, toda obra cuyas acciones presenten modificaciones de acuerdo con registros históricos o conocimiento previo dado a través de juicio de expertos, que se encuentren interdependientes entre sí, presentará una variación expresada en términos cuantitativos que será equivalente a la suma de las varianzas de las actividades involucradas en su ruta crítica (Barraza, Back y Mata, 2004; Hazır, 2015) y, por tanto, comprensible por medio de la aplicación de la AVG y las curvas $S$ estocásticas. A partir de lo anterior, se definió la línea base del proyecto a través de tres parámetros:

- Mínimo: cálculo realizado por medio de la consideración del valor de media obtenido menos una desviación estándar. 
- Medio: cálculo realizado por medio de la consideración del valor de media obtenido.

- Máximo: cálculo realizado por medio de la consideración del valor de media obtenido más una desviación estándar.
En la figura 4, se observa el área que compone la varianza natural de la obra y, por tanto, se infiere que una correcta gestión del director del proyecto se presenta siempre y cuando en la aplicación de un AVG se sitúen los componentes de $(\mathrm{AC})$ valor gastado y $(\mathrm{EV})$ valor ganado en las áreas formadas por el cruce entre líneas.

Figura 4. Curvas de línea base y flujos de caja de acuerdo con las consideraciones mínimo, medio y máximo

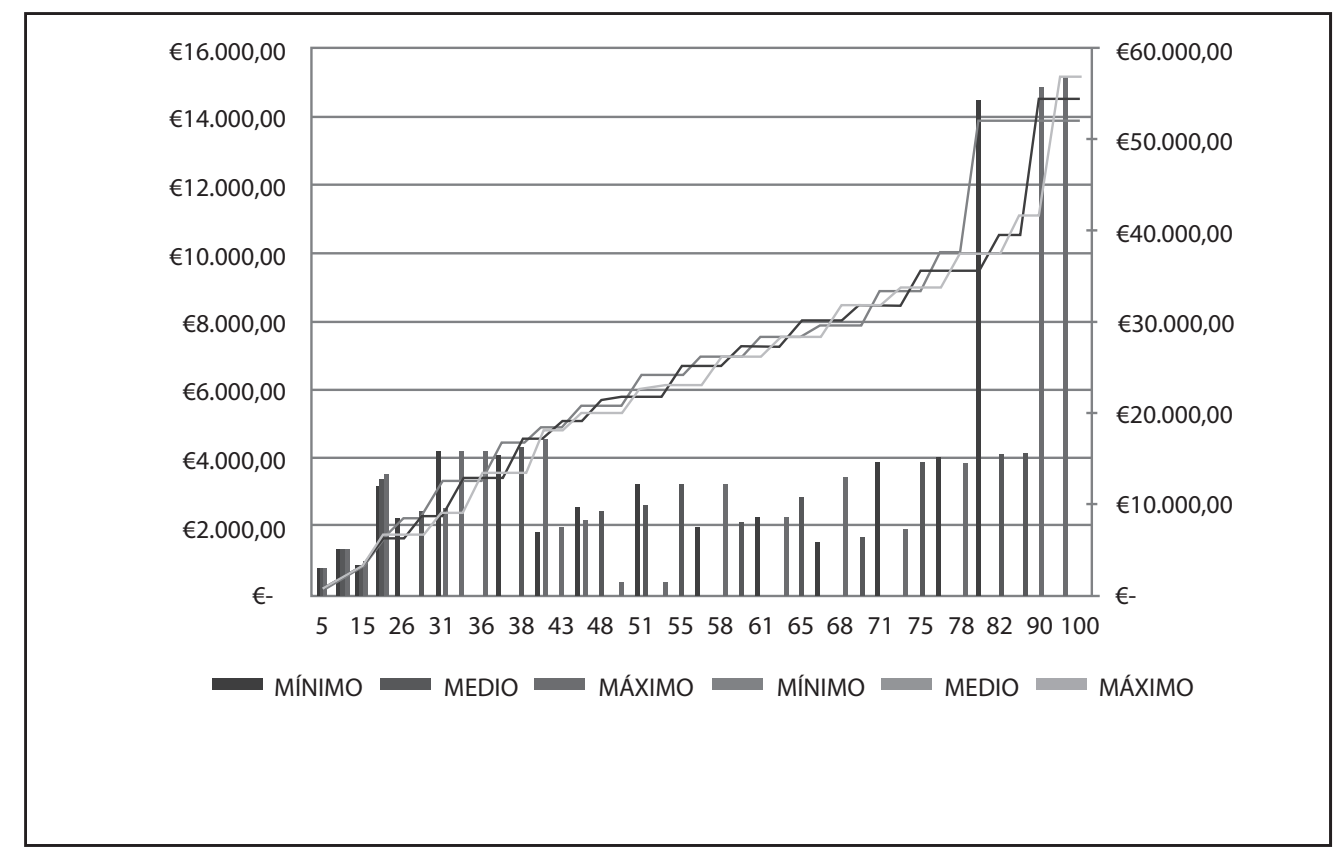

Fuente. Elaboración propia.

Con el objetivo de verificar la hipótesis anterior, se procede a determinar los valores de valor gastado y valor ganado en un proyecto que presentase reprocesos en las actividades mencionadas. Para ello, se realizó la aplicación del modelo Monte Carlo, por medio de 10000 simulaciones aplicando como supuestos de entrada los valores históricos de las variables y como pronósticos de salida las medias determinadas a través de los ajustes de distribución; para esto, los parámetros de las distribuciones se definieron a partir de los estadísticos calculados en el punto 5 (figuras 5, 6 y 7). 
Figura 5. Resultados del modelo de Monte Carlo, Actividad aislamiento de techo 1.1.5

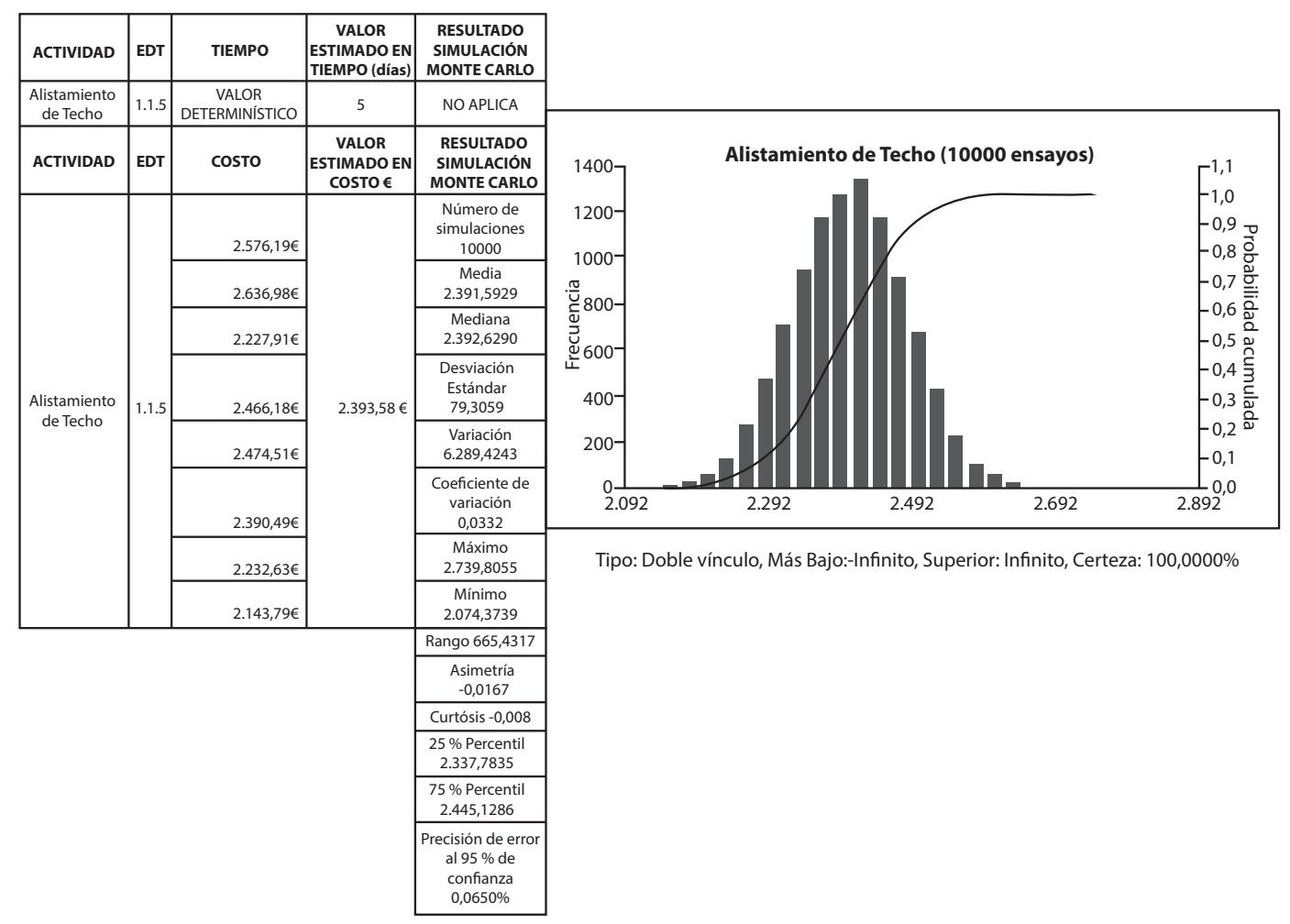

Fuente. Elaboración propia.

Figura 6. Resultados del modelo de Monte Carlo, Actividad red eléctrica, 2. ${ }^{a}$ etapa 1.2.7

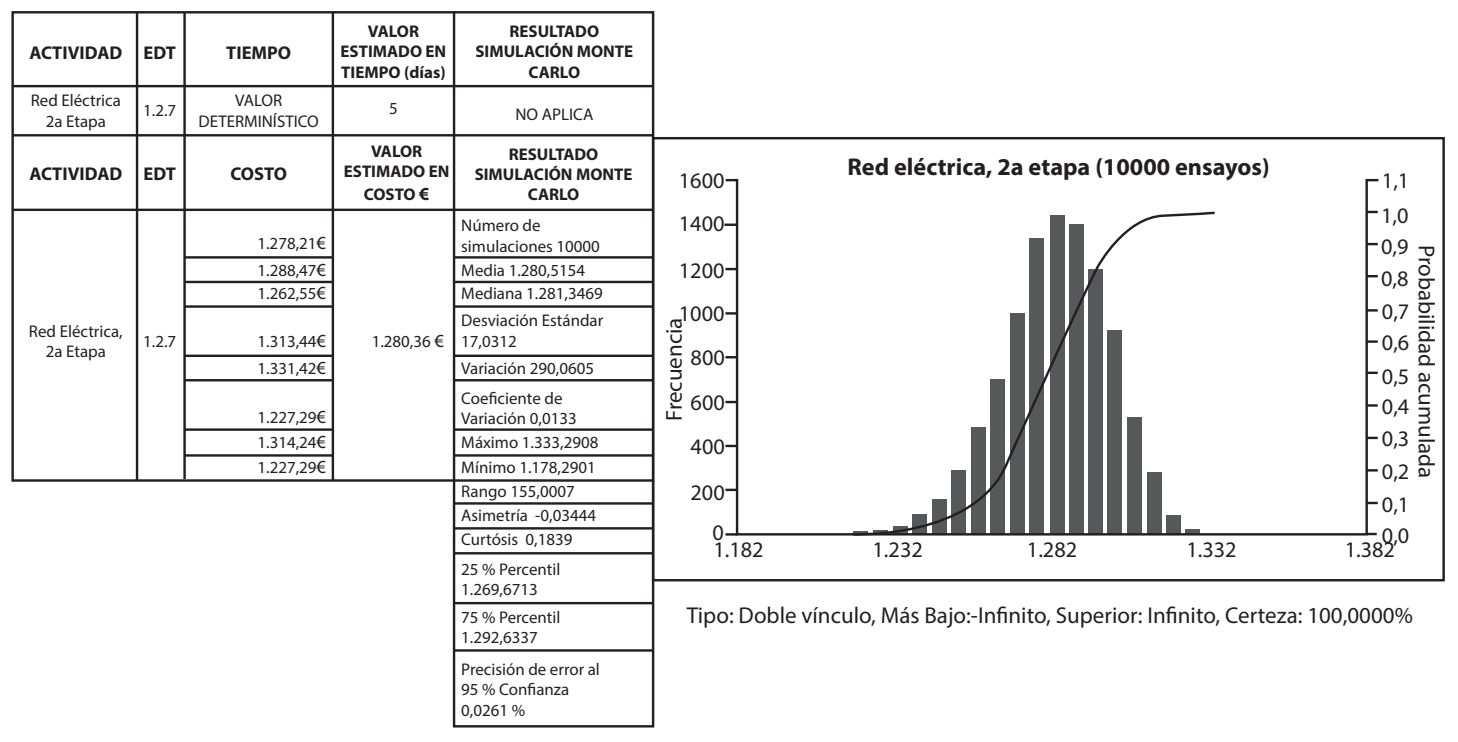

Fuente. Elaboración propia. 
Figura 7. Resultados del modelo de Monte Carlo, Actividad finalización de sistema eléctrico 1.4.2

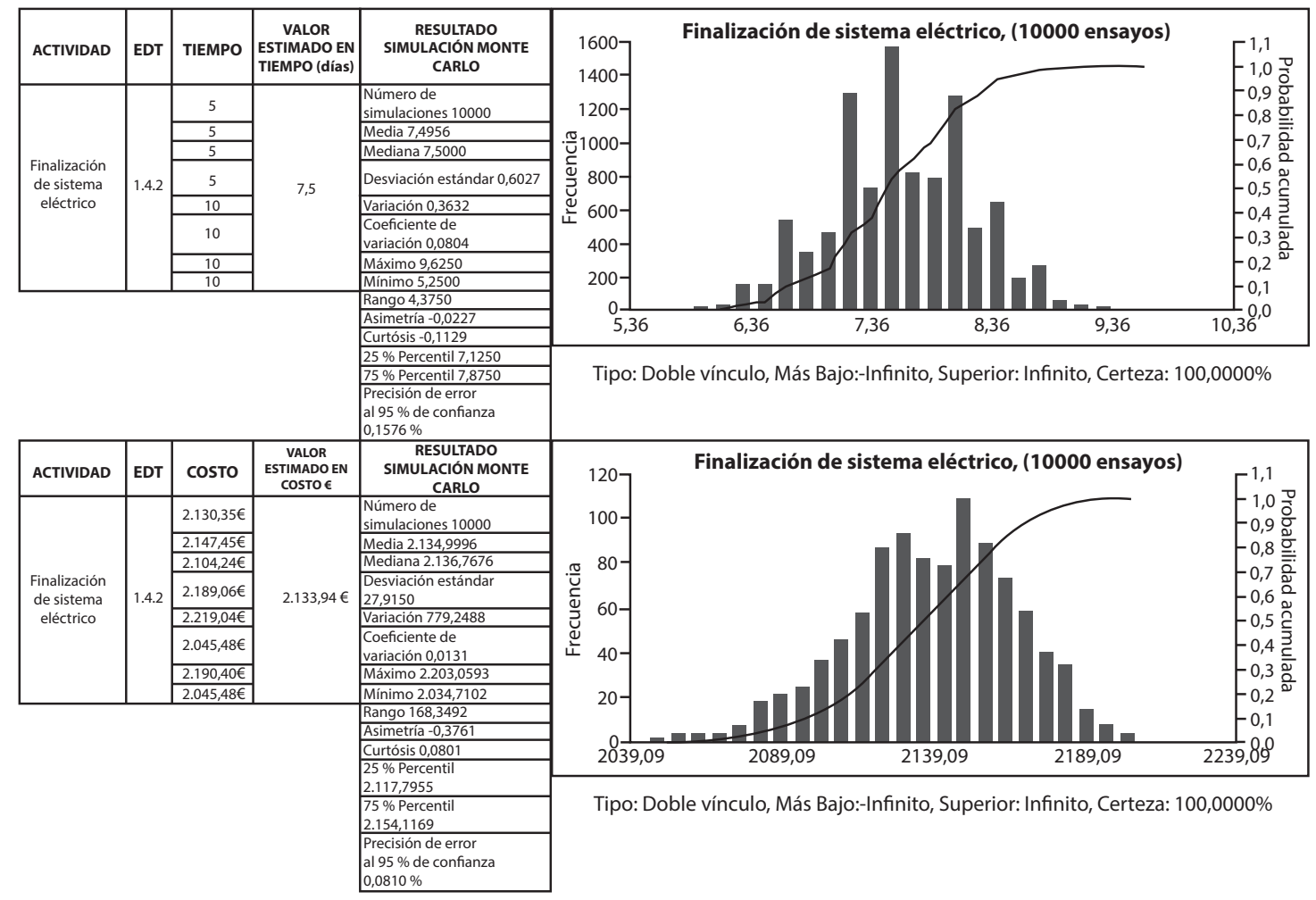

Fuente. Elaboración propia.

Finalizados los cálculos a través del modelo de Monte Carlo, se procede a construir una nueva tabla donde se identifican los valores de los parámetros tiempo y costo para los valores de media, percentil 25 y $75 \%$ calculados (tabla 3 ).

Los valores del percentil $25 \%$ y del percentil $75 \%$ se tomaron con el objetivo de permitir la visualización de ajustes en la figura de tal forma que eviten ser reconocidos como valores atípicos en el momento de comparar los gráficos de curva $S$ generados en la aplicación de la técnica de valor ganado. Para esto, fueron graficados los valores de costo acumulado en cada uno de los casos y comparados con las curvas de línea base determinadas en numerales anteriores (figura 8). 
Tabla 3. Listado de actividades clasificadas con los valores de media, percentil $25 \%$ y percentil $75 \%$.

\begin{tabular}{|c|c|c|c|c|c|c|c|}
\hline & & \multicolumn{3}{|c|}{ TIEMPO (días) } & \multicolumn{3}{|c|}{ COSTO EUR } \\
\hline ACTIVIDAD & EDT & MEDIA & $\begin{array}{c}\text { PERCENTIL } \\
25 \%\end{array}$ & $\begin{array}{c}\text { PERCENTIL } \\
75 \%\end{array}$ & MEDIA & $\begin{array}{c}\text { PERCENTIL } \\
25 \%\end{array}$ & $\begin{array}{c}\text { PERCENTIL } \\
75 \%\end{array}$ \\
\hline Sistema de ventilación & 1.1 .1 & 5 & 5 & 5 & 772,50 EUR & 772,50 EUR & 772,50 EUR \\
\hline san / cv, 1a Etapa & 1.1 .2 & 5 & 5 & 5 & $1.288,55$ EUR & $1.288,55$ EUR & $1.288,55$ EUR \\
\hline Red eléctrica, 1a Etapa & 1.1 .3 & 5 & 5 & 5 & 853,89 EUR & 846,74 EUR & 861,95 EUR \\
\hline Sistema de Hidráulico & 1.1 .4 & 5 & 5 & 5 & 960,00 EUR & 960,00 EUR & 960,00 EUR \\
\hline Aislamiento de Techo & 1.1 .5 & 5 & 5 & 5 & 2.391,59 EUR & $2.337,78$ EUR & $2.445,12$ EUR \\
\hline Aplicaciones de Yeso & 1.1 .6 & 8,75 & 8,75 & 8,75 & 2.393,58 EUR & 2.393,58 EUR & 2.393,58 EUR \\
\hline $\begin{array}{l}\text { Instalación de red de } \\
\text { gas }\end{array}$ & 1.2 .1 & 5 & 5 & 5 & 2.231,25 EUR & 2.231,25 EUR & 2.231,25 EUR \\
\hline san / cv, 2a Etapa & 1.2 .2 & 5 & 5 & 5 & 1.932,83 EUR & 1.932,83 EUR & $1.932,83$ EUR \\
\hline Sistema de ventilación & 1.2 .3 & 5 & 5 & 5 & $1.158,75$ EUR & $1.158,75$ EUR & $1.158,75$ EUR \\
\hline Aislamiento de piso & 1.2 .4 & 5 & 5 & 5 & 6.251,61 EUR & 3.058,01 EUR & 3.283,94 EUR \\
\hline Enchape metálico & 1.2 .5 & 5 & 5 & 5 & 1.955,31 EUR & $1.955,31$ EUR & $1.955,31$ EUR \\
\hline Pintura de interior & 1.2 .6 & 5 & 5 & 5 & 1.132,06 EUR & 1.132,06 EUR & $1.132,06$ EUR \\
\hline Red eléctrica, 2a Etapa & 1.2 .7 & 5 & 5 & 5 & 1.280,51 EUR & $1.269,67$ EUR & 1.292,63 EUR \\
\hline $\begin{array}{l}\text { Instalación de servicios } \\
\text { (energía, agua, gas) }\end{array}$ & 1.2 .8 & 1,5 & 1,5 & 1,5 & 318,75 EUR & 318,75 EUR & 318,75 EUR \\
\hline san / cv, 3a Etapa & 1.3 .1 & 5 & 5 & 5 & 3.221,38 EUR & 3.221,38 EUR & 3.221,38 EUR \\
\hline Piso & 1.3 .2 & 5 & 5 & 5 & 2.085,82 EUR & $2.085,82$ EUR & 2.085,82 EUR \\
\hline Escaleras & 1.3 .3 & 5 & 5 & 5 & 2.816,77 EUR & 2.816,77 EUR & 2.816,77 EUR \\
\hline Carpintería interior & 1.3 .4 & 5 & 5 & 5 & 1.698,09 EUR & 1.698,09 EUR & 1.698,09 EUR \\
\hline Acabados de cocina & 1.3 .5 & 5 & 5 & 5 & 3.825,00 EUR & 3.825,00 EUR & $3.825,00$ EUR \\
\hline $\begin{array}{l}\text { Finalización de Sistema } \\
\text { de ventilación }\end{array}$ & 1.4 .1 & 7,5 & 7,5 & 7,5 & 1.931,25 EUR & $1.931,25$ EUR & $1.931,25$ EUR \\
\hline $\begin{array}{l}\text { Finalización de Sistema } \\
\text { Eléctrico }\end{array}$ & 1.4 .2 & 7,49 & 7,12 & 7,87 & 2.134,99 EUR & 2.117,79 EUR & 2.154,11 EUR \\
\hline Finalización de san /cv & 1.4 .3 & 7,5 & 7,5 & 7,5 & 6.442,75 EUR & 6.442,75 EUR & 6.442,75 EUR \\
\hline Finalización de piso & 1.4 .4 & 7,49 & 7,12 & 7,87 & 5.621,52 EUR & $5.103,76$ EUR & 5.473,63 EUR \\
\hline $\begin{array}{l}\text { Finalización de } \\
\text { acabados de yeso }\end{array}$ & 1.4 .5 & 7,5 & 7,5 & 7,5 & 3.191,44 EUR & 3.191,44 EUR & 3.191,44 EUR \\
\hline
\end{tabular}

Fuente. Elaboración propia. 
Figura 8. Curvas de valor gastado del proyecto

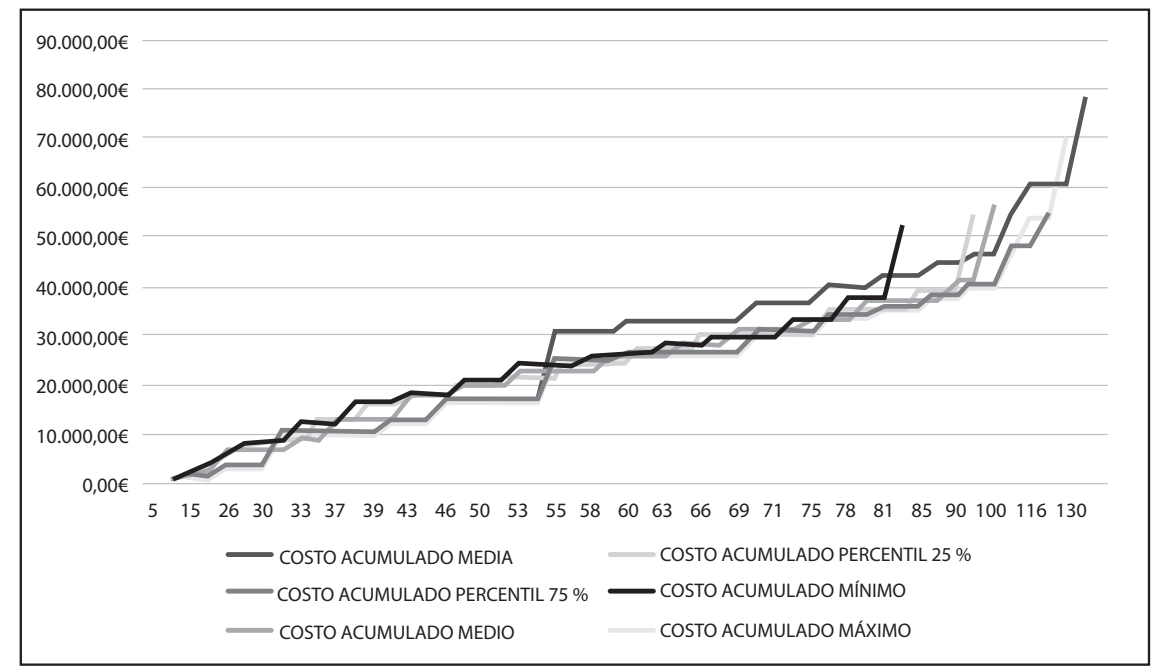

Fuente. Elaboración propia.

De acuerdo con los datos arrojados, se aplica la técnica de valor ganado, con el objetivo de comprender las condiciones de desarrollo del proyecto y validar el método. Para esto, se realiza el análisis de las curvas de valor planeado
(PV) obtenidas de los parámetros mínimos, medios y máximos y se comparan con las curvas de valor gastado $(\mathrm{AC})$ y valor ganado $(\mathrm{EV})$ del percentil $25 \%$, media y percentil $75 \%$ (figuras 9, 10 y 11).

Figura 9. Curvas S estocásticas, análisis respecto de valores mínimos

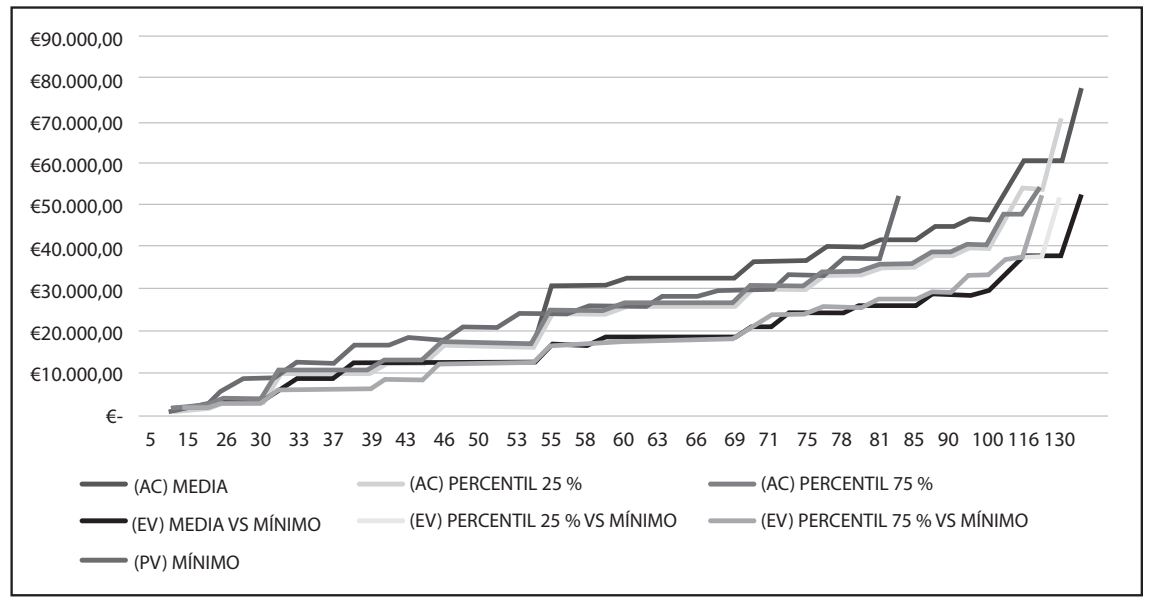

Fuente. Elaboración propia. 
Figura 10. Curvas S estocásticas, análisis respecto de valores medios

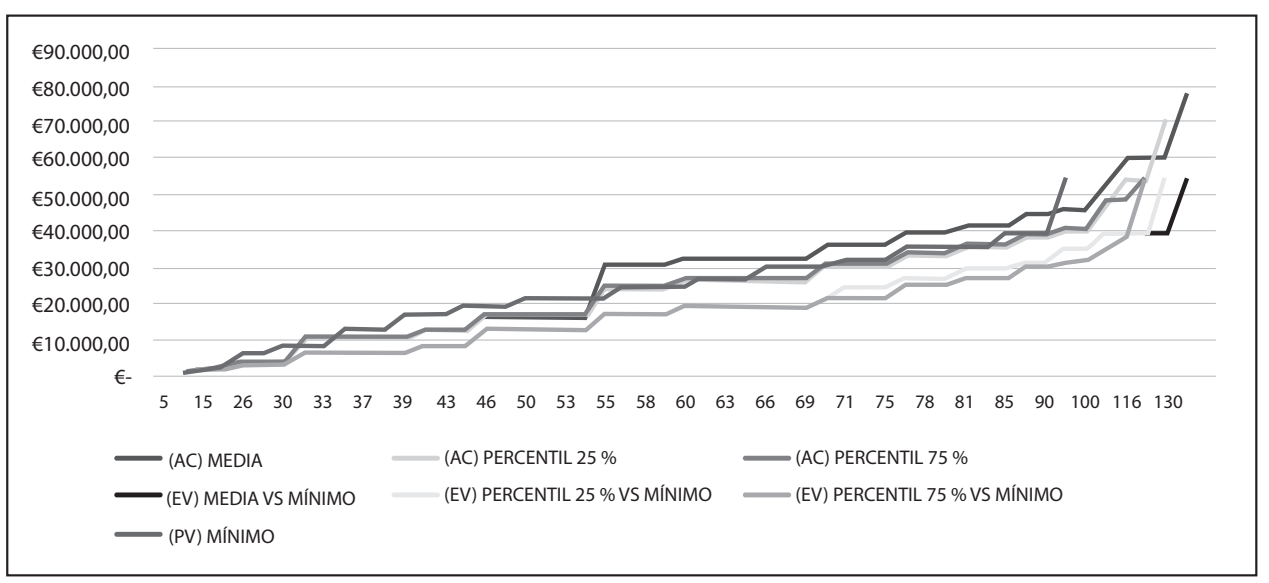

Fuente. Elaboración propia.

Figura 11. Curvas $S$ estocásticas, análisis respecto de valores máximos

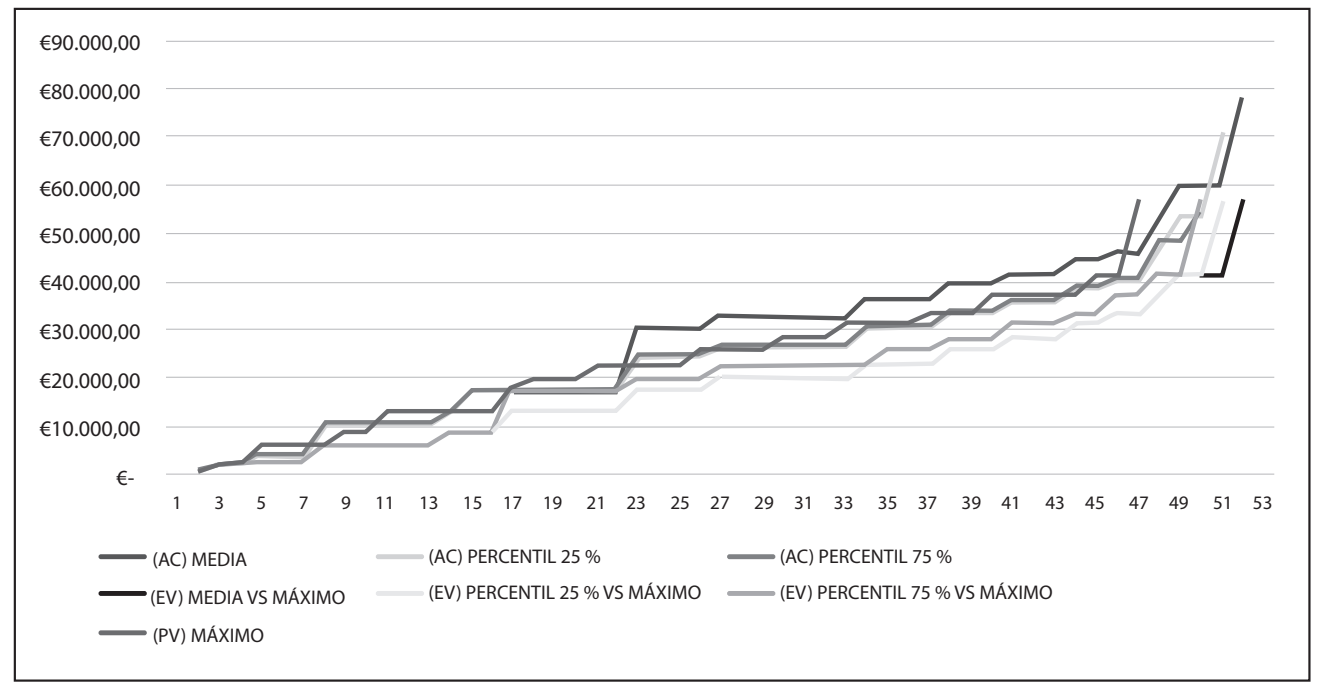

Fuente. Elaboración propia.

Con los datos arrojados en las figuras, se de variación del programa (SV), variación del realiza la aplicación de las fórmulas de valor ganado, tomando como parámetros de análisis las fechas de finalización de cada una de las fases del proyecto y determinando los valores costo $(\mathrm{CV})$, índice de desempeño de los costos (CPI), índice de desempeño del tiempo (SPI) e índice de desempeño para completar (TCPI) (tablas 4, 5 y 6). 
Tabla 4. Indicadores valor ganado respecto de valores mínimos

\begin{tabular}{|c|c|c|c|c|c|c|c|}
\hline \multirow{2}{*}{$\begin{array}{l}\text { Parámetro de } \\
\text { Referencia }\end{array}$} & \multirow{2}{*}{$\begin{array}{l}\text { Punto de } \\
\text { Análisis }\end{array}$} & \multirow{2}{*}{$\begin{array}{c}\text { Días } \\
\text { Trabajados }\end{array}$} & \multirow{2}{*}{$\begin{array}{c}\text { Valor } \\
\text { Planeado (pv) }\end{array}$} & \multicolumn{4}{|c|}{ Valor ganado (ev) } \\
\hline & & & & $\begin{array}{l}\text { Percentil } \\
25 \%\end{array}$ & Media & $\begin{array}{r}\text { Perc } \\
75\end{array}$ & $\begin{array}{l}\text { ntil } \\
\%\end{array}$ \\
\hline \multirow{4}{*}{$\begin{array}{l}\text { Valor mínimo } \\
\text { de costo } \\
\text { acumulado }\end{array}$} & $\begin{array}{l}\text { Inicio etapa de } \\
\text { Acabados }\end{array}$ & 26 & $8.280,83 €$ & $2.888,35 €$ & $2.888,35 €$ & \multicolumn{2}{|c|}{$2.888,35 €$} \\
\hline & Fase 2 & 46 & $20.815,71 €$ & $12.444,91 €$ & $12.444,91 €$ & \multicolumn{2}{|c|}{$12.444,91 €$} \\
\hline & Fase 3 & 71 & $33.473,50 €$ & $24.037,09 €$ & $24.037,09 €$ & \multicolumn{2}{|c|}{$24.037,09 €$} \\
\hline & Acabado final & 81 & $51.937,50 €$ & $28.178,65 €$ & $25.969,21 €$ & \multicolumn{2}{|c|}{$28.178,65 €$} \\
\hline \multirow{2}{*}{$\begin{array}{l}\text { Parámetro de } \\
\text { Referencia }\end{array}$} & \multirow{2}{*}{$\begin{array}{l}\text { Punto de } \\
\text { Análisis }\end{array}$} & \multicolumn{3}{|c|}{ Valor gastado (ac) } & \multicolumn{3}{|c|}{ Variación del programa (sv) } \\
\hline & & $\begin{array}{l}\text { Percentil } \\
25 \%\end{array}$ & Media & $\begin{array}{l}\text { Percentil } \\
75 \%\end{array}$ & $\begin{array}{l}\text { Percentil } \\
25 \%\end{array}$ & Media & $\begin{array}{l}\text { Percentil } \\
75 \%\end{array}$ \\
\hline \multirow{4}{*}{$\begin{array}{l}\text { Valor mínimo } \\
\text { de costo } \\
\text { acumulado }\end{array}$} & $\begin{array}{l}\text { Inicio etapa de } \\
\text { acabados }\end{array}$ & $3.754,53 €$ & $3.768,83 €$ & $3.784,95 €$ & $-5.392,47$ & $-5.392,47 €$ & $-5.392,47 €$ \\
\hline & Fase 2 & $16.907,75 €$ & $17.029,67 €$ & $17.152,85 €$ & $-8.370,80 €$ & $-8.370,80 €$ & $-8.370,80 €$ \\
\hline & Fase 3 & $30.127,99 €$ & $36.658,79 €$ & $30.870,87 €$ & $-9.436,41 €$ & $-9.436,41 €$ & $-9.436,41 €$ \\
\hline & Acabado final & $35.435,19 €$ & $41.965,99 €$ & $36.178,07 €$ & $-23.758,86 €$ & $-25.968,29 €$ & $-23.758,86 €$ \\
\hline \multirow{2}{*}{$\begin{array}{l}\text { Parámetro de } \\
\text { Referencia }\end{array}$} & \multirow{2}{*}{$\begin{array}{l}\text { Punto de } \\
\text { Análisis }\end{array}$} & \multicolumn{3}{|c|}{ Variación del costo $(\mathrm{cv})$} & \multicolumn{3}{|c|}{$\begin{array}{l}\text { Índice de desempeño del } \\
\text { Tiempo (spi) }\end{array}$} \\
\hline & & $\begin{array}{l}\text { Percentil } \\
25 \%\end{array}$ & Media & $\begin{array}{l}\text { Percentil } \\
75 \%\end{array}$ & $\begin{array}{l}\text { Percentil } \\
25 \%\end{array}$ & Media & $\begin{array}{l}\text { Percentil } \\
75 \%\end{array}$ \\
\hline \multirow{4}{*}{$\begin{array}{l}\text { Valor mínimo } \\
\text { de costo } \\
\text { acumulado }\end{array}$} & $\begin{array}{l}\text { Inicio etapa de } \\
\text { acabados }\end{array}$ & $-866,18 €$ & $-880,48 €$ & $-896,60 €$ & 0,35 & 0,35 & 0,35 \\
\hline & Fase 2 & $-4.462,85 €$ & $-4.584,77 €$ & $-4.707,95 €$ & 0,60 & 0,60 & 0,60 \\
\hline & Fase 3 & $-6.090,90 €$ & $-12.621,70 €$ & $-6.833,78 €$ & 0,72 & 0,72 & 0,72 \\
\hline & Acabado final & $-7.256,55 €$ & $-15.996,78 €$ & $-7.999,43 €$ & 0,54 & 0,50 & 0,54 \\
\hline \multirow{2}{*}{$\begin{array}{l}\text { Parámetro de } \\
\text { Referencia }\end{array}$} & \multirow{2}{*}{$\begin{array}{l}\text { Punto de } \\
\text { Análisis }\end{array}$} & \multicolumn{3}{|c|}{$\begin{array}{l}\text { Indice de desempeño de } \\
\text { Los costos (cpi) }\end{array}$} & \multicolumn{3}{|c|}{$\begin{array}{l}\text { Indice de desempeño para } \\
\text { Completar (tcpi) }\end{array}$} \\
\hline & & $\begin{array}{l}\text { Percentil } \\
25 \%\end{array}$ & Media & $\begin{array}{l}\text { Percentil } \\
75 \%\end{array}$ & $\begin{array}{l}\text { Percentil } \\
25 \%\end{array}$ & Media & $\begin{array}{l}\text { Percentil } \\
75 \%\end{array}$ \\
\hline \multirow{4}{*}{$\begin{array}{c}\text { Valor mínimo } \\
\text { de costo } \\
\text { acumulado }\end{array}$} & $\begin{array}{l}\text { Inicio etapa de } \\
\text { acabados }\end{array}$ & 0,77 & 0,77 & 0,76 & 1,02 & 1,02 & 1,02 \\
\hline & Fase 2 & 0,74 & 0,73 & 0,73 & 1,13 & 1,13 & 1,14 \\
\hline & Fase 3 & 0,80 & 0,66 & 0,78 & 1,28 & 1,83 & 1,32 \\
\hline & Acabado final & 0,80 & 0,62 & 0,78 & 1,44 & 2,60 & 1,51 \\
\hline
\end{tabular}

Fuente. Elaboración propia. 
Tabla 5. Indicadores valor ganado respecto de valores medios

\begin{tabular}{|c|c|c|c|c|c|c|c|}
\hline \multirow{2}{*}{$\begin{array}{l}\text { Parámetro de } \\
\text { Referencia }\end{array}$} & \multirow{2}{*}{$\begin{array}{l}\text { Punto de } \\
\text { Análisis }\end{array}$} & \multirow{2}{*}{$\begin{array}{c}\text { Días } \\
\text { Trabajados }\end{array}$} & \multirow{2}{*}{$\begin{array}{c}\text { Valor } \\
\text { Planeado } \\
\text { (pv) }\end{array}$} & \multicolumn{4}{|c|}{ Valor ganado (ev) } \\
\hline & & & & $\begin{array}{c}\text { Percentil } \\
25 \%\end{array}$ & Media & $\begin{array}{r}\text { Perc } \\
75\end{array}$ & $\begin{array}{l}\text { entil } \\
\%\end{array}$ \\
\hline \multirow{4}{*}{$\begin{array}{l}\text { Valor mínimo } \\
\text { de costo } \\
\text { acumulado }\end{array}$} & $\begin{array}{c}\text { Inicio etapa de } \\
\text { Acabados }\end{array}$ & 28 & $8.661,79 €$ & $2.914,63 €$ & $2.914,63 €$ & \multicolumn{2}{|c|}{$2.914,63 €$} \\
\hline & Fase 2 & 50 & $21.799,84 €$ & $12.825,87 €$ & $12.825,87 €$ & \multicolumn{2}{|c|}{$12.825,87 €$} \\
\hline & Fase 3 & 75 & $35.446,90 €$ & $27.107,04 €$ & $25.021,22 €$ & \multicolumn{2}{|c|}{$25.021,22 €$} \\
\hline & Acabado final & 90 & $54.360,83 €$ & $35.446,90 €$ & $31.621,90 €$ & \multicolumn{2}{|c|}{$31.621,90 €$} \\
\hline \multirow{2}{*}{$\begin{array}{l}\text { Parámetro de } \\
\text { Referencia }\end{array}$} & \multirow{2}{*}{$\begin{array}{l}\text { Punto de } \\
\text { Análisis }\end{array}$} & \multicolumn{3}{|c|}{ Valor gastado (ac) } & \multicolumn{3}{|c|}{ Variación del programa (sv) } \\
\hline & & $\begin{array}{c}\text { Percentil } \\
25 \%\end{array}$ & Media & $\begin{array}{c}\text { Percentil } \\
75 \%\end{array}$ & $\begin{array}{c}\text { Percentil } \\
25 \%\end{array}$ & Media & $\begin{array}{c}\text { Percentil } \\
75 \%\end{array}$ \\
\hline \multirow{4}{*}{$\begin{array}{l}\text { Valor mínimo } \\
\text { de costo } \\
\text { acumulado }\end{array}$} & $\begin{array}{c}\text { Inicio etapa de } \\
\text { acabados }\end{array}$ & $3.754,53 €$ & $3.768,83 €$ & $3.784,95 €$ & $-5.747,17 €$ & $-5.747,17 €$ & $-5.747,17 €$ \\
\hline & Fase 2 & $16.907,75 €$ & $17.029,67 €$ & $17.152,85 €$ & $-8.973,97 €$ & $-8.973,97 €$ & $-8.973,97 €$ \\
\hline & Fase 3 & $33.349,37 €$ & $39.880,17 €$ & $34.092,25 €$ & $-8.339,86 €$ & $-10.425,68 €$ & $-10.425,68 €$ \\
\hline & Acabado final & $39.950,05 €$ & $46.480,85 €$ & $40.692,93 €$ & $-18.913,93 €$ & $-22.738,93 €$ & $-22.738,93 €$ \\
\hline \multirow{2}{*}{$\begin{array}{l}\text { Parámetro de } \\
\text { Referencia }\end{array}$} & \multirow{2}{*}{$\begin{array}{l}\text { Punto de } \\
\text { Análisis }\end{array}$} & \multicolumn{3}{|c|}{ Variación del costo $(\mathrm{cv})$} & \multicolumn{3}{|c|}{$\begin{array}{c}\text { Índice de desempeño del } \\
\text { Tiempo (spi) }\end{array}$} \\
\hline & & $\begin{array}{c}\text { Percentil } \\
25 \%\end{array}$ & Media & $\begin{array}{c}\text { Percentil } \\
75 \%\end{array}$ & $\begin{array}{c}\text { Percentil } \\
25 \%\end{array}$ & Media & $\begin{array}{l}\text { Percentil } \\
75 \%\end{array}$ \\
\hline \multirow{4}{*}{$\begin{array}{l}\text { Valor mínimo } \\
\text { de costo } \\
\text { acumulado }\end{array}$} & $\begin{array}{c}\text { Inicio etapa de } \\
\text { acabados }\end{array}$ & $-839,91 €$ & $-854,21 €$ & $-870,33 €$ & 0,34 & 0,34 & 0,34 \\
\hline & Fase 2 & $-4.081,88 €$ & $-4.203,80 €$ & $-4.326,98 €$ & 0,59 & 0,59 & 0,59 \\
\hline & Fase 3 & $-6.242,33 €$ & $-14.858,95 €$ & $-9.071,03 €$ & 0,76 & 0,71 & 0,71 \\
\hline & Acabado final & $-4.503,15 €$ & $-14.858,95 €$ & $-9.071,03 €$ & 0,65 & 0,58 & 0,58 \\
\hline \multirow{2}{*}{$\begin{array}{l}\text { Parámetro de } \\
\text { Referencia }\end{array}$} & \multirow{2}{*}{$\begin{array}{l}\text { Punto de } \\
\text { Análisis }\end{array}$} & \multicolumn{3}{|c|}{$\begin{array}{l}\text { Indice de desempeño de } \\
\text { Los costos (cpi) }\end{array}$} & \multicolumn{3}{|c|}{$\begin{array}{l}\text { Indice de desempeño para } \\
\text { Completar (tcpi) }\end{array}$} \\
\hline & & $\begin{array}{l}\text { Percentil } \\
25 \%\end{array}$ & Media & $\begin{array}{l}\text { Percentil } \\
75 \%\end{array}$ & $\begin{array}{l}\text { Percentil } \\
25 \%\end{array}$ & Media & $\begin{array}{l}\text { Percentil } \\
75 \%\end{array}$ \\
\hline \multirow{4}{*}{$\begin{array}{l}\text { Valor mínimo } \\
\text { de costo } \\
\text { acumulado }\end{array}$} & $\begin{array}{l}\text { Inicio etapa de } \\
\text { acabados }\end{array}$ & 0,78 & 0,77 & 0,77 & 1,02 & 1,02 & 1,02 \\
\hline & Fase 2 & 0,76 & 0,75 & 0,75 & 1,11 & 1,11 & 1,12 \\
\hline & Fase 3 & 0,81 & 0,63 & 0,73 & 1,30 & 2,03 & 1,45 \\
\hline & Acabado final & 0,89 & 0,68 & 0,78 & 1,31 & 2,89 & 1,66 \\
\hline
\end{tabular}

Fuente. Elaboración propia. 
Tabla 6. Indicadores valor ganado respecto de valores máximos

\begin{tabular}{|c|c|c|c|c|c|c|c|}
\hline \multirow{2}{*}{$\begin{array}{l}\text { Parámetro de } \\
\text { Referencia }\end{array}$} & \multirow{2}{*}{$\begin{array}{l}\text { Punto de } \\
\text { Análisis }\end{array}$} & \multirow{2}{*}{$\begin{array}{c}\text { Días } \\
\text { Trabajados }\end{array}$} & \multirow{2}{*}{$\begin{array}{c}\text { Valor } \\
\text { Planeado } \\
\text { (pv) }\end{array}$} & \multicolumn{4}{|c|}{ Valor ganado $(\mathrm{ev})$} \\
\hline & & & & $\begin{array}{l}\text { Percentil } \\
25 \%\end{array}$ & Media & $\begin{array}{r}\text { Perc } \\
7\end{array}$ & $\begin{array}{l}\text { ntil } \\
\%\end{array}$ \\
\hline \multirow{4}{*}{$\begin{array}{l}\text { Valor mínimo } \\
\text { de costo } \\
\text { acumulado }\end{array}$} & $\begin{array}{l}\text { Inicio etapa de } \\
\text { Acabados }\end{array}$ & 31 & $9.042,76 €$ & $6.471,83 €$ & $6.471,83 €$ & \multicolumn{2}{|c|}{$6.471,83 €$} \\
\hline & Fase 2 & 53 & $22.783,98 €$ & $13.206,84 €$ & $13.206,84 €$ & \multicolumn{2}{|c|}{$17.724,87 €$} \\
\hline & Fase 3 & 78 & $37.420,31 €$ & $26.005,36 €$ & $26.005,36 €$ & \multicolumn{2}{|c|}{$28.244,87 €$} \\
\hline & Acabado final & 99 & $56.784,16 €$ & $33.595,31 €$ & $33.595,31 €$ & \multicolumn{2}{|c|}{$37.420,31 €$} \\
\hline \multirow{2}{*}{$\begin{array}{l}\text { Parámetro de } \\
\text { Referencia }\end{array}$} & \multirow{2}{*}{$\begin{array}{l}\text { Punto de } \\
\text { Análisis }\end{array}$} & \multicolumn{3}{|c|}{ Valor gastado $(\mathrm{ac})$} & \multicolumn{3}{|c|}{ Variación del programa (sv) } \\
\hline & & $\begin{array}{l}\text { Percentil } \\
25 \%\end{array}$ & Media & $\begin{array}{l}\text { Percentil } \\
\quad 75 \%\end{array}$ & Percentil $25 \%$ & Media & $\begin{array}{l}\text { Percentil } \\
\quad 75 \%\end{array}$ \\
\hline \multirow{4}{*}{$\begin{array}{l}\text { Valor mínimo } \\
\text { de costo } \\
\text { acumulado }\end{array}$} & $\begin{array}{l}\text { Inicio etapa de } \\
\text { acabados }\end{array}$ & $10.350,09 €$ & $10.472,01 €$ & $10.595,19 €$ & $-2.570,93 €$ & $-2.570,93 €$ & $-2.570,93 €$ \\
\hline & Fase 2 & $16.907,75 €$ & $17.029,67 €$ & $24.879,48 €$ & $-9.577,13 €$ & $-9.577,13 €$ & $-5.059,10 €$ \\
\hline & Fase 3 & $33.349,37 €$ & $39.880,17 €$ & $34.092,25 €$ & $-11.414,95 €$ & $-11.414,95 €$ & $-9.175,43 €$ \\
\hline & Acabado final & $39.950,05 €$ & $46.480,85 €$ & $40.692,93 €$ & $-23.188,85 €$ & $-23.188,85 €$ & $-19.363,85 €$ \\
\hline \multirow{2}{*}{$\begin{array}{l}\text { Parámetro de } \\
\text { Referencia }\end{array}$} & \multirow{2}{*}{$\begin{array}{l}\text { Punto de } \\
\text { Análisis }\end{array}$} & \multicolumn{3}{|c|}{ Variación del costo $(\mathrm{cv})$} & \multicolumn{3}{|c|}{$\begin{array}{l}\text { Índice de desempeño del } \\
\text { Tiempo (spi) }\end{array}$} \\
\hline & & $\begin{array}{l}\text { Percentil } \\
25 \%\end{array}$ & Media & $\begin{array}{l}\text { Percentil } \\
75 \%\end{array}$ & Percentil $25 \%$ & Media & $\begin{array}{l}\text { Percentil } \\
75 \%\end{array}$ \\
\hline \multirow{4}{*}{$\begin{array}{l}\text { Valor mínimo } \\
\text { de costo } \\
\text { acumulado }\end{array}$} & $\begin{array}{l}\text { Inicio etapa de } \\
\text { acabados }\end{array}$ & $-3.878,26 €$ & $-4.000,18 €$ & $-4.123,36 €$ & 0,72 & 0,72 & 0,72 \\
\hline & Fase 2 & $-3.700,91 €$ & $-3.822,83 €$ & $-7.154,61 €$ & 0,58 & 0,58 & 0,78 \\
\hline & Fase 3 & $-7.344,01 €$ & $-13.874,81 €$ & $-5.847,38 €$ & 0,69 & 0,69 & 0,75 \\
\hline & Acabado final & $-6.354,74 €$ & $-12.885,54 €$ & $-3.272,62 €$ & 0,59 & 0,59 & 0,66 \\
\hline \multirow{2}{*}{$\begin{array}{l}\text { Parámetro de } \\
\text { Referencia }\end{array}$} & \multirow{2}{*}{$\begin{array}{l}\text { Punto de } \\
\text { Análisis }\end{array}$} & \multicolumn{3}{|c|}{$\begin{array}{l}\text { Indice de desempeño de } \\
\text { Los costos (cpi) }\end{array}$} & \multicolumn{3}{|c|}{$\begin{array}{l}\text { Indice de desempeño para } \\
\text { Completar (tcpi) }\end{array}$} \\
\hline & & $\begin{array}{l}\text { Percentil } \\
25 \%\end{array}$ & Media & $\begin{array}{l}\text { Percentil } \\
75 \%\end{array}$ & Percentil $25 \%$ & Media & $\begin{array}{l}\text { Percentil } \\
75 \%\end{array}$ \\
\hline \multirow{4}{*}{$\begin{array}{l}\text { Valor mínimo } \\
\text { de costo } \\
\text { acumulado }\end{array}$} & $\begin{array}{l}\text { Inicio etapa de } \\
\text { acabados }\end{array}$ & 0,63 & 0,62 & 0,61 & 1,08 & 1,09 & 1,09 \\
\hline & Fase 2 & 0,78 & 0,78 & 0,71 & 1,09 & 1,10 & 1,22 \\
\hline & Fase 3 & 0,78 & 0,65 & 0,83 & 1,31 & 1,82 & 1,26 \\
\hline & Acabado final & 0,84 & 0,72 & 0,92 & 1,38 & 2,25 & 1,20 \\
\hline
\end{tabular}

Fuente. Elaboración propia. 


\section{Conclusiones}

Dor medio de la metodología y experimentación realizada en el estudio, se demostró el impacto de los costos generados por concepto de reproceso, fruto de fallas o falencias desde el punto de vista de la calidad. Para el caso presentado, se asume que este reproceso requiere volver a realizarse $\mathrm{y}$, por tanto, se hace necesario causar nuevamente los valores y tiempos definidos. Para ello, se presenta un análisis sobre cada una de las figuras de curvas de valor gastado del proyecto, el cual se representa en la figura 8 , que fue elaborada con la aplicación de la simulación de Monte Carlo; en ella se evidencia que disminuyó 10000 escenarios posibles. Los valores generados por reprocesos pueden resultar bajo un percentil del $25 \%$, un tiempo de desarrollo total de la obra de 125 días, un costo total del proyecto de EUR 70 417,15 y el percentil $75 \%$ un tiempo total de 129 días a un costo total de EUR 54 582,4. Finalmente, el tiempo y los costos medios son de 131 días y EUR 78 017,87, y las curvas S estocásticas, generadas en la planeación del proyecto y utilizadas como herramientas de control y toma de decisiones en su ejecución, presentan rangos de finalización del proyecto de 82 días a 99 días, con un cálculo total de entre EUR 51 937,5 a EUR 56 784,16; esta percepción permite comprender el impacto de los costos de calidad desde el punto de vista del cumplimiento del presupuesto y del cronograma estimados.

En segunda instancia, y a partir de la aplicación de la EVM, es posible determinar que los costos de variación del programa (SV) y variación del costo $(\mathrm{CV})$ de las tablas 4,5 y 6 evidencian retrasos y sobrecostos, debido a su coste negativo. Por su parte, el índice de desempeño del costo (CPI) y el índice de desempeño del tiempo (SPI) reflejan la eficiencia desde el punto de vista de los costos y el tiempo, respectivamente, las cuales demuestran valores inferiores al 100 $\%$, que alcanzan hasta un $34 \%$ en la primera fase de los análisis realizados respecto de los valores mínimos y medios.

En el estudio, no es evidente la recuperación de los costos de calidad descritos a lo largo del proyecto bajo ninguna de las opciones analizadas - percentil $25 \%$, percentil $75 \%$ y media-, por lo que se evidencia la necesidad de ser tenidos presente en el presupuesto de los proyectos.

Los resultados generaron un impacto negativo sobre los índices de desempeño para completar (TCPI). Este indicador es viable expresarlo como la mejora desde el punto de vista de la eficiencia que se debe aplicar a la obra si se desea finalizar en el tiempo y costo definido a partir de los escenarios mínimos, medios y máximos mencionados, tras lo cual se encontraron valores de hasta 2,89 equivalente al $189 \%$, en el caso del paralelo de la curva media con relación a la planeación mínima, y de 1,20, equivalente al $20 \%$ en la comparación de la curva media respecto de la planeación máxima. Esto traduce que, aun en una planeación con escenarios pesimistas soportada estadísticamente bajo la suma de las varianzas de las actividades que componen la ruta crítica, no es posible establecer reservas de contingencias suficientes que permitan blindar el proyecto de los costos de calidad, que en caso de presentarse pueden afectar directamente la línea base del proyecto (Navarro, 2006). 
Es primordial destacar que los datos históricos obtenidos son pocos, por tanto, es posible encontrar variaciones importantes respecto de la realidad debido al tipo de distribución de probabilidad ajustada. Cabe resaltar que, de acuerdo con las cantidades observadas desde el punto de vista del tiempo, fueron utilizadas distribuciones discretas, mientras que en las variables en torno a costo se realizó el análisis a través de distribuciones continuas. Lo anterior teniendo en cuenta que la forma de control del tiempo en los proyectos analizados demostró ser por días, tomando estos como números enteros. Sin embargo, en casos en los que la programación del proyecto permita el manejo de cifras decimales en el parámetro tiempo, se considera estadísticamente factible el uso de las distribuciones continuas para este atributo.

La aplicación de las diferentes distribuciones a los parámetros de tiempo y costo permite explicar mejor el comportamiento de las variables, y con ello generar estimaciones válidas desde el componente estadístico; deducciones que facilitan la proyección de los costos futuros. Con ellos se siembra la base para la implementación de algoritmos genéticos que permitan obtener resultados fiables en evaluaciones de proyectos generadas en etapas tempranas, lo que la toma de decisiones y la optimización de los recursos en las organizaciones.

La generación de las líneas base bajo los parámetros mínimos, medios y máximos permite observar el rango de labor en el que puede desarrollarse un director de proyectos, lo que mejora la toma de decisiones en etapas de realización, situación que se considera compleja de entender bajo el desarrollo de curvas $\mathrm{S}$ determinísticas. Además, la aplicación del modelo de Monte Carlo admite la estimación de 10000 escenarios posibles, así como la determinación de rangos de trabajo a través de la inclusión del percentil $25 \%$ y del percentil $75 \%$, lo que demuestra las posibles variaciones adicionales que pueden presentarse, fruto de ajustes o cambios generados debido a reprocesos. Esto hace posible establecer con mejor claridad elementos de pronóstico durante la ejecución del proyecto.

La aplicación de la EVM admite validar los resultados obtenidos, ya que se realizan observaciones que demuestran variaciones importantes desde el punto de vista del tiempo y de los costos. Cabe resaltar que esta técnica permite la integración de los componentes de alcance y calidad a través del supuesto de que se refiere a que dichos componentes pueden ser expresados respecto del tiempo y del costo.

Por otra parte, es primordial destacar la importancia del almacenamiento de información histórica de proyectos, ya que con ella es posible generar nuevas teorías, métodos y demás elementos en pro del avance del conocimiento científico. Es recomendable la construcción de una base de datos sobre información de obras de diversos tipos bajo el contexto nacional, con la finalidad de ajustar futuras investigaciones a entornos cercanos, y con ello fortalecer el dominio respecto de la dirección de proyectos a través de una relación más estrecha entre universidad y empresa.

Para finalizar, se sugiere continuar con investigaciones en este campo de trabajo, donde se permita la inclusión de componentes tales como el impacto de las interdependencias entre actividades con reprocesos ubicados en la ruta crítica y fuera de ella, así como la aplicación de experimentaciones en torno a metodologías de dirección como gestión de proyectos por cadena crítica (CCPM - Critical chain project management), que se enfoca en la construcción de cadenas críticas y la generación de colchones de alimentación y de proyecto en la programación del proyecto, con el fin de evitar el impacto de estas

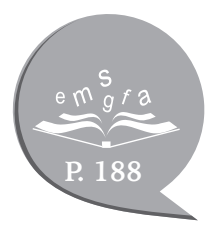


eventualidades. Así mismo, se exhorta a la inserción del concepto de números difusos, con el fin de observar el comportamiento y definir de forma más exacta los pronósticos elaborados. Por último, se recomienda la repetición de la experimentación en obras relacionadas con otras áreas de conocimiento, con la finalidad de extender el uso de la metodología utilizada y validarla a través de múltiples experimentaciones.

\section{Referencias}

Ahmed, S. M., Aoieong, R. T., Tang, S. L. y Zheng, D. X. (2005). A comparison of quality management systems in the construction industries of Hong Kong and the USA. International Journal of Quality \& Reliability Management, 22(2), 149-161.

Barraza, G. A., Back, W. E. y Mata, F. (2004). Probabilistic forecasting of project performance using stochastic $S$ curves. Journal of Construction Engineering and Management, $130(1), 25-32$.

Batselier, J. y Vanhoucke, M. (2015). Construction and evaluation framework for a real-life project database. International Journal of Project Management, 33(3), 697-710.

Bernal, C. (2016). Metodología de la investigación (4. ${ }^{\mathrm{a}}$ ed.). México: Pearson.

Campanella, J. (1997). Fundamentos de los costos de calidad, lineamientos y práctica. México: McGraw-Hill.

Climent Serrano, S. (2004). Los costes de calidad como estrategia empresarial: evidencia empírica en la Comunidad Valenciana (Tesis doctoral, Universitat de València, España). Recuperado de https://dialnet.unirioja.es/ servlet/dctes? codigo $=6843$

Gido, J. y Clements, J. (2012). Administración exitosa de proyectos $\left(5 .^{\mathrm{a}}\right.$ ed.). México: Cengage Learning.

Gracia Villar, S. y Dzul López, L. A. (2007). Modelo PEF de costes de la calidad como herramienta de gestión en empresas constructoras: una visión actual. Revista Ingeniería de Construcción, 22(1), 43-56.
Hazır, Ö. (2015). A review of analytical models, approaches and decision support tools in project monitoring and control. International Journal of Project Management, 33(4), 808-815.

Lledó, P. y Rivarola, G. (2007). Gestión de proyectos. Buenos Aires: Pearson Educación.

Navarro, D. (2006). Seguimiento de proyectos con el análisis del valor ganado. Recuperado de http://www.armell. com/docs/avg_v1.pdf

Project Management Institute. (2017). Guía de los fundamentos para la dirección de proyectos: Guía del PMBOK (6. ${ }^{\mathrm{a}}$ ed.). Newtown Square: Project Management Institute.

Rincón, L. (2012). Introducción a los procesos estocásticos. México: Universidad Nacional Autónoma de México.

Vanhoucke, M., Coelho, J. y Batselier, J. (2016). An overview of project data for integrated project management and control. Journal of Modern Project Management, 3(2), 6-21.

Zapata, C. J., Piñeros, L. C. y Castaño, D. A. (2004). El método de simulación de Monte Carlo en estudios de confiabilidad de sistemas de distribución de energía eléctrica. Scientia et Technica, 1(24).

Zapata, V. J. (2011, abril 11). Gerencia de proyectos [Entrada blog]. Recuperado de http://ceresegp.blogspot. com/2011/04/valor-monetario-esperado.html 